\title{
Treatment of Tachycardia and
} Bradycardia in a Persistent Left Superior Vena Cava Patient Who Underwent

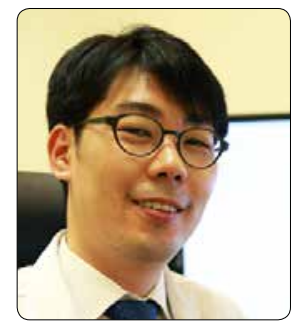
Warden's Procedure and Tricuspid Annuloplasty

Youngjin Cho, MD

Department of Internal Medicine, Seoul National University Bundang Hospital, Seongnam, Republic of Korea

Received: February 2, 2016

Revision Received: March 8, 2016

Accepted: March 28, 2016

Correspondence: Youngjin Cho, MD

Department of Internal Medicine, Seoul National

University Bundang Hospital, Gumi-ro 173 Beon-

gil, Bundang-gu, Seongnam-si, Gyeonggi-do,

Republic of Korea, 13620

Tel: 82-31-787-7083 Fax: 82-31-787-4051

E-mail: cho_y@snubh.org

Copyright $(2016$ The Official Journal of Korean Heart Rhythm Society Editorial Board \& MMK Co., Ltd.

\begin{abstract}
A 53-year-old female was admitted to our institution with alternating atrial flutter and junctional bradycardia. The patient had undergone the Warden procedure to correct sinus venosus type atrial septal defect combined with partial anomalous pulmonary venous return, and ring tricuspid annuloplasty for severe tricuspid regurgitation. She also had persistent left superior vena cava (PLSVC). With the assistance of a 3D electroanatomic mapping system, catheter ablation therapy was used successfully to treat atrial flutter associated with a channel in the right atrial scar, and a pacemaker was implanted through the PLSVC because of resulting symptomatic bradycardia.
\end{abstract}

Key Words: - Congenital Heart Defect - Cardiac Surgical Procedures -Atrial Flutter -Catheter Ablation -Artificial Pacemaker

\section{Introduction}

Symptomatic supraventricular tachycardia is a common indication for radiofrequency catheter ablation treatment. Often, supraventricular tachycardia is associated with sick sinus syndrome, which requires the implantation of a permanent pacemaker. Underlying structural heart diseases such as complex congenital anomalies or a history of prior cardiac surgery can hinder successful procedural treatment for these arrhythmias. We report a case of tachycardia and bradycardia that was complicated by several congenital cardiac anomalies and a prior cardiac surgery, and that was treated successfully.

\section{Case}

A 53-year-old female patient was admitted to the emergency room because of chest discomfort. The patient had undergone a workup for dyspnea on exertion 8 years ago, which revealed sinus venosus type atrial septal defect (ASD), combined with partial anomalous pulmonary venous return (PAPVR). The patient underwent the Warden procedure at that time, involving transection of the superior vena cava (SVC), extension of ASD with intra-atrial baffling of the PAPVR to the left atrium, and anastomosis of the transected SVC to the right atrial appendage (Figure 1). In addition, ring tricuspid annuloplasty was performed because of severe tricuspid regurgitation, and a diagnosis of 

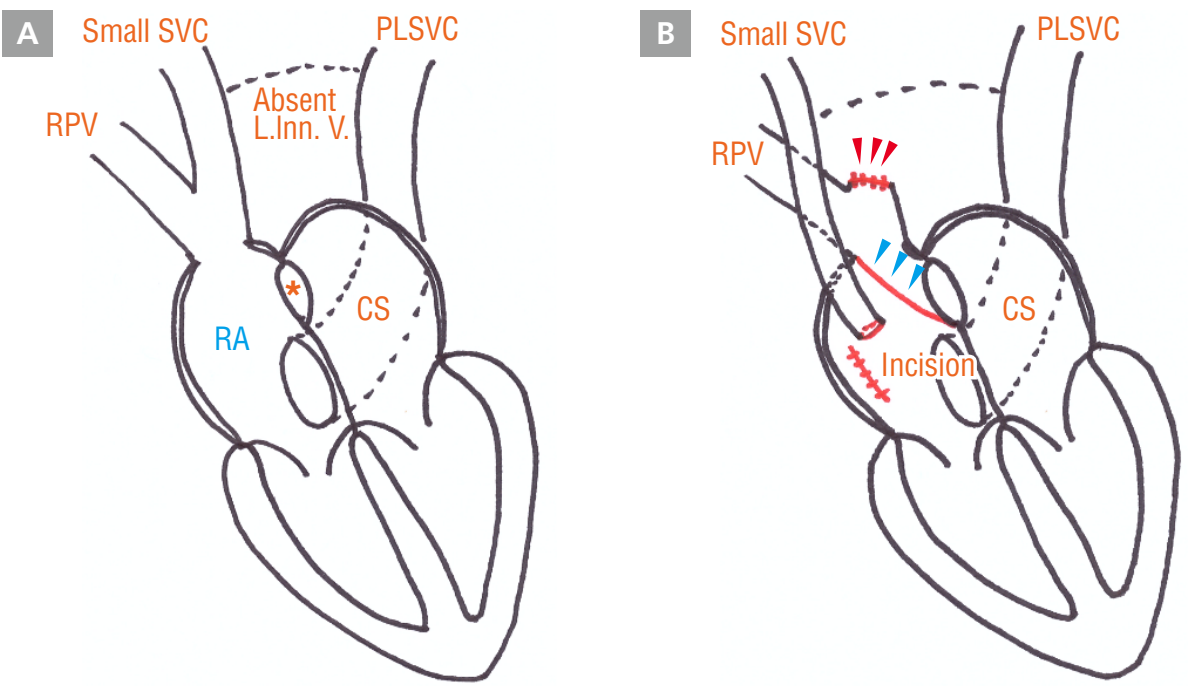

Figure 1. Illustrations of $(A)$ before and $(B)$ after the Warden procedure in this case. The asterisk indicates the sinus venosus type atrial septal defect (ASD). The small right SVC was transected just above the anomalous draining from the RPV (red arrowheads), and anastomosed to the RA appendage. The ASD was extended with a patch (blue arrowheads) to connect the anomalous PV return to the left atrium.

CS, coronary sinus; L. Inn. V, left innominate vein; PLSVC, persistent left superior vena cava; RA, right atrium; RPV,

right pulmonary vein; SVC, superior vena cava.

concomitant persistent left superior vena cava (PLSVC) without the left innominate vein was made. Atrial flutter developed 2 weeks after the cardiac surgery, and because of palpitations, the patient began taking flecainide ( $50 \mathrm{mg}$ twice daily) 6 months prior to the emergency room visit mentioned in this report. Electrocardiograms taken in the emergency room showed alternating atrial flutter and marked sinus bradycardia with junctional escape beats (Figure 2). The patient's symptom was therefore associated with both tachycardia and bradycardia. Laboratory findings, including cardiac enzymes, were within the normal range, and the echocardiogram showed a normal left ventricular ejection fraction (56\%). Transesophageal echocardiography excluded the existence of left atrial thrombus and the residual ASD shunt flow. Cardiac computed tomography revealed a prominent PLSVC, and there was no evidence of any change since the last cardiac surgery.

Since cessation of flecainide did not improve the patient's symptoms, radiofrequency catheter ablation (RFCA) for atrial flutter was performed. Electroanatomic mapping was obtained using the Ensite NavX system (St. Jude Medical), and suggested a dual-loop figure- 8 macro-reentry atrial flutter (tachycardia cycle length, $245 \mathrm{msec}$ ) associated with a channel between right atrial scars around the crista terminalis, which might have been produced by the atriotomy during the previous cardiac surgery (Figure 3). RF energy was delivered to the atrium around the channel, and atrial flutter was terminated. RF ablation was additionally performed to achieve bidirectional cavotricuspid isthmus (CTI) block, because CTI-dependent typical atrial flutter was induced during the post-RFCA electrophysiologic study. After that, the atrial tachyarrhythmia was no longer inducible.

However, symptomatic bradycardia persisted and the patient underwent permanent pacemaker implantation. Since the left innominate vein was absent and the right SVC that was anastomosed to the right atrial appendage was too small for pacemaker leads, the leads were introduced to the right atrium and ventricle via PLSVC (Figure 4). Following dual-chamber pacemaker implantation (Advisa DR MRI, Medtronic), the patient's symptoms were clear

\section{Discussion}

During development, cardinal veins first form a symmetrical 
A

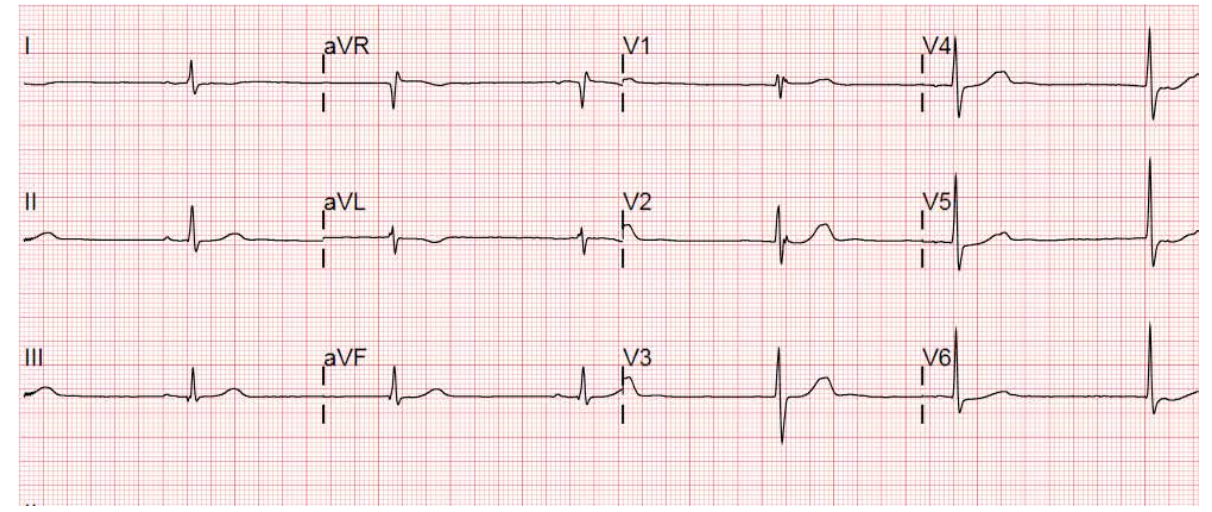

B

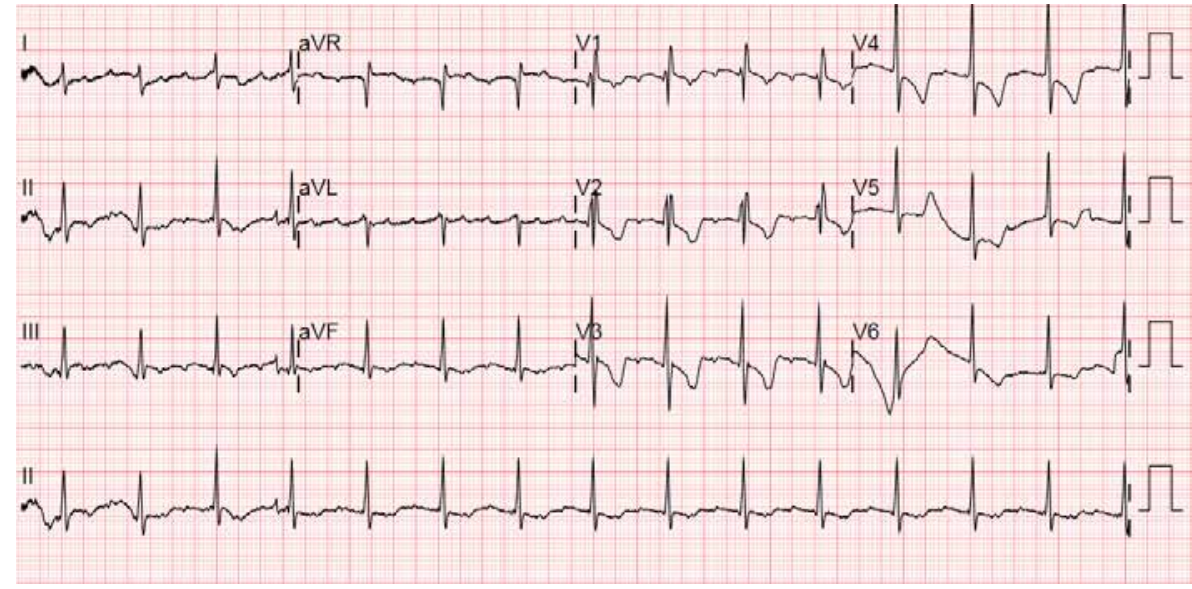

Figure 2. Electrocardiograms taken at the emergency department showing alternating (A) atrial flutter and (B) marked sinus bradycardia with junctional escape beats.

A

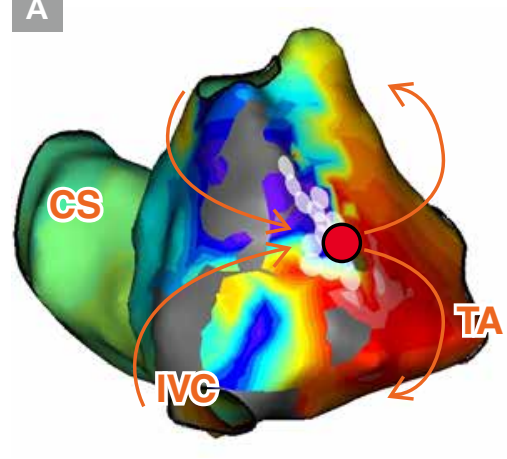

B

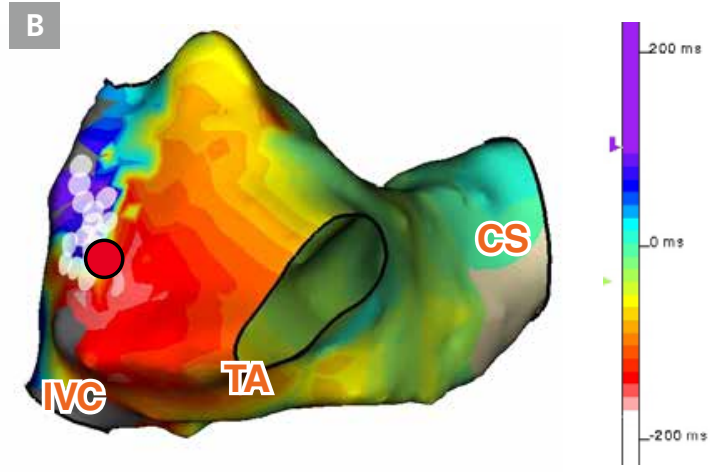

Figure 3. Right atrial activation map of atypical atrial flutter. (A) Right lateral and (B) AP view. AP, anteroposterior; CS, coronary sinus; IVC, inferior vena cava; TA, tricuspid annulus. 


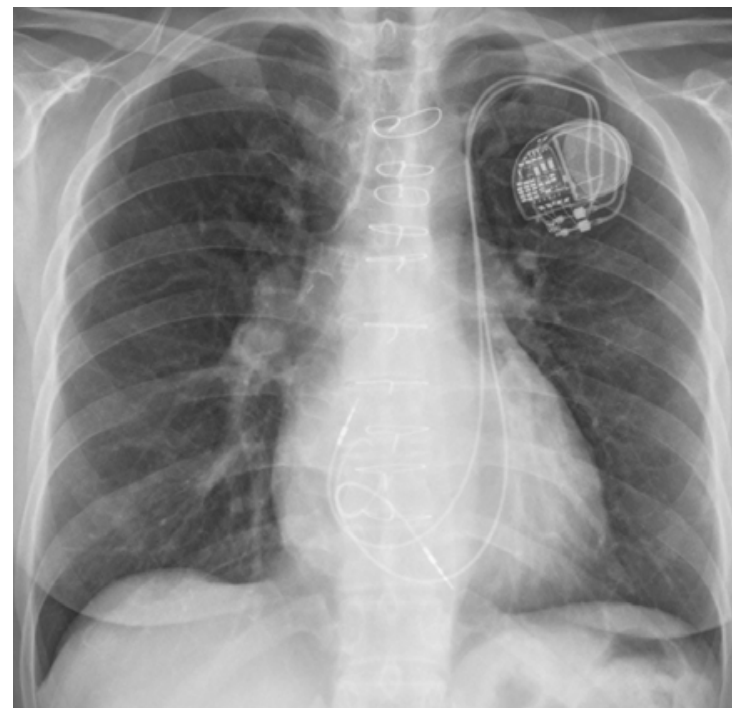

B

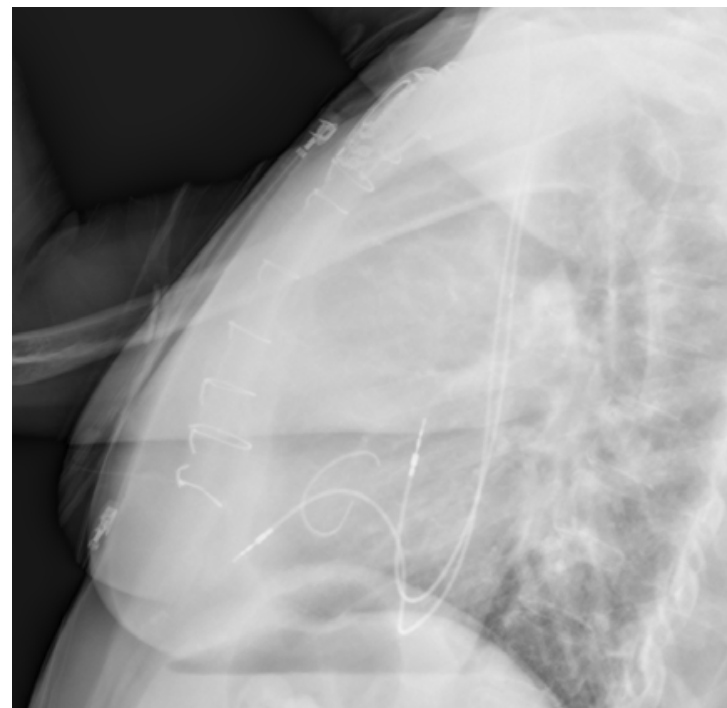

Figure 4. Chest X-ray after the pacemaker implantation through the left persistent superior vena cava. (A) posterioranterior (PA) and (B) left lateral view.

system, and then posterior cardinal veins join together to drain into the sinus horn. Normally, the superior vena cava develops on the right side, while the left cardinal vein regresses and forms the ligament of the left vena cava. The left common cardinal vein and the left horn of the sinus venosus form the coronary sinus. A persistent left superior vena cava develops when the left anterior cardinal vein and the left common cardinal vein do not regress. ${ }^{1}$ The majority of patients with PLSVC are asymptomatic and usually PLSVC is an innocent finding with $0.5 \%$ prevalence in the general population. ${ }^{2,3}$ However, PLSVC may cause arrhythmia in some patients because the dilated coronary sinus is adjacent to the left-sided primitive pacemaking tissue. ${ }^{4,5}$

The patient in this case had sinus venosus ASD and PAPVR, in addition to PLSVC. Although atrial septal defect is a relatively common adult congenital heart disease, sinus venosus ASD is a rare type that leads to interatrial communication outside the fossa ovalis due to maldevelopment of the atrial infolding that normally separates the two atria in the area between the SVC and the right pulmonary veins. ${ }^{6}$ Only about 10 to $15 \%$ of ASD cases are accompanied by anomalous drainage of the right pulmonary veins to the SVC, and this combined congenital anomaly accounts for less than $1 \%$ of total congenital heart disease cases. ${ }^{7}$ Sinus venosustype ASD accompanied by both PAPVR and PLSVC is very rare, and only several cases have been reported. ${ }^{8}$

In this case, the patient presented with atrial tachyarrhythmia.
The flutter waves in the inferior leads of the 12-lead ECG mimicked typical counterclockwise atrial flutter, but an inverted flutter wave in the V1 lead suggested atypical flutter. Electroanatomic mapping using the Ensite 3D system showed macro-reentrant tachycardia through the channel between the right atrial scars around the crista terminalis. Considering that the patient had undergone a Warden procedure 8 years ago for the correction of sinus venosus type ASD and PAPVR, it seems probable that the scar corresponded to the site of the prior atriotomy. However, there is still the possibility that the scar and the channel formed spontaneously. Stevenson et al. ${ }^{9}$ reported that $2.8 \%(8 / 286)$ of macroreentrant atrial tachycardia cases in their medical center were associated with spontaneous scarring in the right atrium, especially around the crista terminalis, and a stable reentry circuit was formed through the channel in the scar in 4 patients (1.4\%). Furthermore, Jais et al. ${ }^{10}$ reported spontaneous left atrial scarring in patients with structural heart diseases. The arrhythmia in this case involved several congenital anomalies and their surgical correction, including tricuspid annuloplasty for severe tricuspid regurgitation. These might have contributed to the abnormal stretching and scarring of the right atrium.

In this case, the bradycardia might also have been caused by the RA scar. Because the Warden procedure does not incise the cavoatrial junction, the risk of sick sinus syndrome is reported to be lower after the Warden procedure than after conventional 
surgery. Nevertheless, fibrosis around the crista terminalis caused symptomatic bradycardia, requiring permanent pacemaker implantation. In a previous study, 5 out of 8 patients with spontaneous RA scar-associated macroreentrant tachycardia eventually underwent permanent pacemaker implantation, as in this case. In addition, PLSVC itself is a known risk factor for sinus node dysfunction. ${ }^{4}$ In the absence of the left innominate vein, and with a small right SVC anastomosed to the right atrial appendage, the pacemaker leads had to be inserted through the left SVC. Though this anatomical variation complicated the procedure, the pacemaker implantation was successful as reported in previous cases. ${ }^{711,12}$

This case presents the successful treatment of both tachycardia and bradycardia in a patient with rare adult congenital anomalies who had previously undergone surgical correction. 3D electroanatomic mapping was useful to reveal the mechanism of the arrhythmia, and a standard dual chamber pacemaker was inserted without difficulty.

\section{References}

1) Sadler TW. Langman's medical embryology. Langman's medical embryology. 2011.

2) Fraser RS, Dvorkin J, Rossall RE, Eidem R. Left superior vena cava: a review of associated congenital heart lesions, catheterization data and roentgenologic findings. Am J Med. 1961;31:711-716.

3) Steinberg I, Dubilier W, Jr., Lukas DS. Persistence of left superior vena cava. Dis Chest. 1953;24:479-488.

4) Morgan DR, Hanratty CG, Dixon LJ, Trimble M, O'Keeffe DB. Anomalies of cardiac venous drainage associated with abnormalities of cardiac conduction system. Europace. 2002;4:281-287.

5) Anderson RH, Latham RA. The cellular architecture of the human atrioventricular node, with a note on its morphology in the presence of a left superior vena cava. J Anat. 1971;109:443-455.

6) Li J, Al Zaghal AM, Anderson RH. The nature of the superior sinus venosus defect. Clin Anat. 1998;11:349-352.

7) Walsh EP, Cecchin F. Arrhythmias in adult patients with congenital heart disease. Circulation. 2007;115:534-545.

8) Benson RE, Songrug T. CT appearance of persistent left superior vena cava, anomalous right superior pulmonary venous return into the right-sided superior vena cava and a sinus venosus-type atrial septal defect. BrJ Radiol. 2009;82:e235-e239.

9) Stevenson IH, Kistler PM, Spence SJ, Vohra JK, Sparks PB, Morton JB, Kalman JM. Scar-related right atrial macroreentrant tachycardia in patients without prior atrial surgery: electroanatomic characterization and ablation outcome. Heart Rhythm. 2005;2:594601.

10) Jais P, Shah DC, Haissaguerre M, Hocini M, Peng JT, Takahashi A, Garrigue S, Le Metayer P, Clementy J. Mapping and ablation of left atrial flutters. Circulation. 2000;101:2928-2934.

11) Westerman GR, Baker J, Dungan WT, Van Devanter SH. Permanent pacing through a persistent left superior vena cava: an approach and report of dual-chambered lead placement. Ann Thorac Surg. 1985;39:174-176.

12) Bae J, Oh S, Kang H, Kim K, Park Y, Choi Y. A Case of Successful Implantation of a DDD Type Permanent Pacemaker in a Patient with Persistent Left Superior and Absent Right Superior Vena Cava. Korean Circ J. 2004;34:623-626. 